\title{
Vascular expression driven by the promoter of a gene encoding a high-affinity potassium transporter HAK5 from Eucalyptus grandis
}

\author{
Carolina S. Costa $^{1} \cdot$ Juliana P. Bravo $^{1}$ - Cíntia L. Ribeiro ${ }^{1,2} \cdot$ Adriana S. Soprano $^{1}$ • \\ Flávio T. Sassaki ${ }^{1,3} \cdot$ Ivan G. Maia ${ }^{1,4}(\mathbb{C}$
}

Received: 11 January 2017 / Accepted: 7 July 2017 / Published online: 14 July 2017

(C) Springer Science+Business Media B.V. 2017

\begin{abstract}
The construction of expression cassettes harboring tissue-specific promoters is a viable alternative to limit transgene expression to specific organs and cell types. In this study, we have functionally characterized the promoter of a Eucalyptus grandis gene encoding a putative high-affinity HAK5-like potassium $\left(\mathrm{K}^{+}\right)$transporter (designated $E g H A K 5$ ) showing root-specific expression. The ability of the EgHAK 5'-flanking region $(\sim 1.3 \mathrm{~kb})$ to drive rootspecific expression of a reporter gene ( $\beta$-glucuronidase; GUS) was examined using transgenic tobacco plants. Histochemical analysis revealed enhanced GUS staining in the vasculature of leaves, hypocotyls and roots, which was also confirmed in histological cross-sections. Moreover, the relative expression of GUS in the roots of the generated transgenic lines was increased in response to $\mathrm{K}^{+}$starvation. Overall, our results indicate that, in a heterologous system, the EgHAK5 promoter shows expression in vascular
\end{abstract}

Communicated by Magda-Viola Hanke.

Data availability The nucleotide sequence data reported in this paper has been submitted to the GenBank database and has been assigned the Accession Number JX276737.

Ivan G. Maia

igmaia@ibb.unesp.br

1 Departamento de Genética, Instituto de Biociências, UNESP, Botucatu, SP 18618-689, Brazil

2 Present Address: Monsanto Company, 700 Chesterfield Pkwy W, Chesterfield, MO 63017, USA

3 Present Address: Instituto de Genética e Bioquímica, Universidade Federal de Uberlândia, Campus Monte Carmelo, Rodovia LMG 746, Km 1 - Bairro Araras, Monte Carmelo, MG, Brazil

4 Departamento de Genética, Instituto de Biociências, UNESP, Botucatu, SP 18618-970, Brazil tissues, mainly within the phloem, and is up-regulated upon potassium deprivation.

Keywords Promoter - Vascular expression - Potassium transporter $\cdot$ Root $\cdot$ Eucalyptus

\section{Introduction}

Promoters are crucial regulatory sequences that enable and control gene expression under specific spatiotemporal conditions. In plants, the recovery of highly active promoters is of major interest especially to improve the control of transgene expression (Hernandez-Garcia and Finer 2014). One useful approach in this regard involves the utilization of tissue-specific promoters that limit transgene expression to a particular organ/tissue, thus avoiding the undesirable effects normally associated with constitutive expression. In this context, genomic resources such as expressed sequence tag (EST) databases represent a prospective source to retrieve candidate genes with desired expression profiles aiming further promoter isolation (Venter and Botha 2004; Hernandez-Garcia and Finer 2014).

In this regard, we have been using the information embedded in publicly available EST libraries as a tool to exploit the Eucalyptus transcriptome and categorize transcripts according to their expression patterns. By using an in silico approach combined with six different statistical tests, we were able to define sets of genes specifically or preferentially expressed in a particular Eucalyptus organ/ tissue type (Vicentini et al. 2005). The retrieved genes constitute a useful repertoire to select tissue-specific candidates for promoter cloning intended for future biotechnological applications in this economically important woody species. 
To date, the number of available promoters in Eucalyptus is limited. Examples include the promoter regions of genes involved in lignin (Lacombe et al. 2000; Lauvergeat et al. 2002; Baghdady et al. 2006; de la Torre et al. 2014) and cellulose ( $\mathrm{Lu}$ et al. 2008; Creux et al. 2008) biosynthesis in Eucalyptus grandis, E. globulus and E. gunnii. In addition, Eucalyptus promoters driving specific expression in roots and in secondary stem, respectively, were also isolated and functionally characterized (Rodrigues et al. 2013; MacMillan et al. 2015). In practice, however, the $35 \mathrm{~S}$ promoter is still the preferred choice for the construction of expression cassettes employed in Eucalyptus (Aggarwal et al. 2015). Therefore, the achievement of confined transgene expression in planta is contingent on the availability of a large number of promoters with appropriate features of organ/tissue specificity.

In the current study, we report the isolation and characterization of the 5'-flanking region of an E. grandis gene validated as specifically expressed in roots. This gene, predicted to encode a high-affinity potassium (HAK) transporter, was selected among those genes showing organ/ tissue-specific expression as determined by the aforementioned in silico approach (Vicentini et al. 2005). Promoter activity of the cloned fragment $(1.3 \mathrm{~kb})$ was evaluated using stably transformed tobacco plants harboring a promoter-reporter gene fusion. Our data indicate that the cloned fragment can drive an inducible reporter expression in vascular tissues, showing great potential as a tool to create transgenic plants.

\section{Materials and methods}

\section{Plant material and growth conditions}

Freshly harvested vegetative and reproductive organs/tissues (root, leaf, stem, vascular cambium, flower bud and fruit) were obtained from $E$. grandis of different ages, grown under greenhouse $\left(28^{\circ} \mathrm{C}, 60 \%\right.$ of relative humidity) or field conditions at Luiz Antônio and Itapetininga, São Paulo, Brazil. The cambial samples were collected from 6-year-old trees essentially as described (Fiorani Celedon et al. 2007), while the leaf samples were obtained from 6-month-old plantlets and from 6- and 3-year-old trees, respectively. All leaf samples were pooled before analysis. For the relative expression analyses, the root and stem samples were taken from plantlets at two developmental stages (1 and 6 months) and from 40-day-old micropropagated seedlings. After harvesting, fresh organ/tissue samples were frozen immediately in liquid nitrogen until RNA extraction. Wild-type (WT) and transgenic tobacco plants (Nicotiana tabacum SR1) were grown in a growth chamber at $22-24^{\circ} \mathrm{C}$ under a $16 \mathrm{~h} / 8 \mathrm{light} /$ dark cycle.

\section{In silico selection of the EST candidate}

The EST candidate (EGMCRT3145G04.g) used in this study was selected from the Brazilian Eucalyptus Genome Sequence Project Consortium (FORESTs) database during a search for the identification of root-specific or root-abundant ESTs. For this, the in silico expression-profile data of Eucalyptus ESTs made available by Vicentini et al. (2005) was employed. Since the selected EST encodes a putative HAK5-like transporter, the nucleotide and deduced amino acid sequences of a previously characterized HAK5 transporter from Arabidopsis thaliana (AtHAK5; Ahn et al. 2004) were employed as queries in subsequent analysis in GenBank (https://www.ncbi.nlm.nih.gov/) and Phytozome v11.0 (http://www.phytozome.net).

\section{RNA extraction and reverse transcription (RT)}

Total RNA was isolated from different organs/tissues of $E$. grandis using a modified CTAB (hexadecyltrimethylammonium bromide) method as described (Korimbocus et al. 2002). Total RNA ( $1 \mu \mathrm{g})$ was treated with RNase-free DNaseI (Fermentas) and reverse-transcribed to cDNA using an oligo-dT17 anchored primer $(2.5 \mathrm{mM})$ and the SuperScript III Reverse Transcriptase Kit (Invitrogen) according to the manufacturer's instructions. All nucleic acid concentrations were measured using a NanoDrop ND1000 Spectrophotometer (NanoDrop Technologies).

\section{Validation of the expression by RT-PCR/qRT-PCR}

To validate the root-specific expression predicted in silico, a qualitative RT-PCR over a panel of different $E$. grandis organs/tissues was performed. Amplification of the target sequence was carried out using Taq polymerase and the following primer pair: VaHAK-F (5'GTGGCGCTGGAGGAA GAAG3') and VaHAK-R (5'GCGACAGAACCCCAAGAA TG3'). In this case, the glyceraldehyde-3-phosphate dehydrogenase (GAPDH) gene from $E$. grandis (primers $5^{\prime} \mathrm{ACC}$ ACGAGCTCAAGGTCAAG3' and 5'ACCACGAGCTCA AGGTCAAG3') was used as endogenous reference control.

The relative expression of the target gene was also examined using quantitative RT-PCR (qRT-PCR). The reactions were performed in a 7500 Real Time PCR System (Applied Biosystems), using the Power SYBR Green Master Mix protocol (Applied Biosystems) and the following genespecific primer pair: EgHAK-F (5'GCCGCGTTGACT CTCTTAACC3') and EgHAK-R (5'AAGAGTGCGCGT GGGACTT3'). The PCR cycling conditions were as follow: $2 \mathrm{~min}$ at $50^{\circ} \mathrm{C}, 10 \mathrm{~min}$ at $95^{\circ} \mathrm{C}$, followed by 45 cycles each of $15 \mathrm{~s}$ at $95^{\circ} \mathrm{C}, 30 \mathrm{~s}$ at $60^{\circ} \mathrm{C}$ and $30 \mathrm{~s}$ at $72^{\circ} \mathrm{C}$. Each reaction was assayed in triplicate in a total volume of $10 \mu \mathrm{l}$, and contained $20 \mathrm{ng}$ of cDNA and $0.2 \mu \mathrm{M}$ of each primer. 
The NADP-dependent isocitrate dehydrogenase gene (specific primers: 5'CTGCTGGAATCTGGTATGAACA3' and 5'TCACTCTGGACATCTCCATCA3') from E. globulus was used as an internal control (Goicoechea et al. 2005).

Cycle threshold $(C t)$ values were obtained for each sample, and relative quantification was determined using the $2^{-\Delta \Delta \mathrm{Ct}}$ method as described (Livak and Schmittgen 2001). Amplification efficiencies were derived from the amplification plots using the program LinRegPCR (Ramakers et al. 2003). A value of two was used in calculations. Relative expression data were analyzed using the Relative Expression Software Tool (REST 2009) and differences with $\mathrm{p}$ values $<0.05$ were considered statistically significant.

\section{Promoter isolation by genome walking (GW), cloning and sequencing}

The 5'flanking region of the candidate gene was amplified using genomic DNA extracted from roots of $E$. grandis using a CTAB method (Lu et al. 2008) and the Universal GenomeWalker Kit (Clontech) following the manufacturer's instructions. For this, two gene-specific primers (HAKGSP1-5'CTTCATGTTTAGCTTGGTCTTCTCC 3' and HAK-GSP2 $\quad-5^{\prime}$ 'GTGATCTCTGACTCTTCTTCTTCC TC3') were designed based on the nucleotide sequence of the starting EST clone. The longest amplification product $(\sim 1.3 \mathrm{~kb})$ obtained was gel-purified, cloned into pGEMTeasy (Promega) and sequenced using the BigDye Terminator v3.1 Sequencing Standard Kit (Applied Biosystems) and an automated ABI Prism 377 sequencer (Applied Biosystems).

For the construction of the expression cassette, the GW-amplified fragment inserted into pGEM-Teasy was re-amplified using $P f u$ DNA polymerase and the primers EgHAK-BamHI (5'CGACGGATCCGCTGGTATA CCGTGA3') and EgHAK-NcoI (5'TATACCATGGTTT TTTCCTCCTCGCC3'), which contain BamHI and NcoI recognition sites (underlined), respectively. The PCRderived product was digested, gel purified and cloned upstream of the promoterless uidA reporter gene (GUS) of the binary vector pCAMBIA-1381z (Cambia), yielding pCAMBIAEgHAKpromo:GUS plasmid.

\section{Generation of transgenic tobacco plants}

The resulting pCAMBIAEgHAKpromo:GUS plasmid was introduced into Agrobacterium tumefaciens LBA4404 and used to transform tobacco ( $N$. tabacum SR1) leaf discs as described (Horsch et al. 1985). Putative transformants were selected in agar plates containing MS (Murashige and Skoog 1962) medium supplemented with hygromycin $(50 \mu \mathrm{g} / \mathrm{ml})$. T-DNA integration in the regenerated lines was confirmed by PCR using genomic DNA extracted from leaves and primers complementary to the amplified promoter and to the GUS coding region (5'GTCTGCCAGTTC AGTTCGTTGTTC3'), respectively. Only self-pollinated $\mathrm{T} 2$ progenies were employed in subsequent assays.

\section{GUS histochemical assays}

Promoter activity was assessed by histochemical GUS staining essentially as described (Jefferson et al. 1987). Seeds of three different positive transgenic lines were germinated in MS medium supplemented with hygromycin $(50 \mu \mathrm{g} / \mathrm{ml})$, and intact 15 -day-old seedlings were sampled for GUS staining using X-Gluc (5-bromo-4-chloro-3indolyl- $\beta$-D-glucuronide). Stained samples were washed in ethanol $70 \%$ for chlorophyll removal and photographed. WT or a transgenic tobacco line bearing the GUS reporter gene under the control of a Cauliflower mosaic virus $35 \mathrm{~S}$ promoter were used as negative and positive controls, respectively. Sections $(20 \mu \mathrm{m})$ of the samples assayed histochemically for GUS activity were obtained using a microtome and processed as previously described (Severino et al. 2012).

\section{Potassium deprivation assays}

Potassium deprivation assays were performed essentially as described (Ahn et al. 2004). Briefly, 2 week-old transgenic tobacco seedlings grown hydroponically in one-fourth strength Hoagland solution (Hoagland and Arnon 1950) were transferred to a $\mathrm{K}^{+}$-free nutrient solution, and sampled after $12 \mathrm{~h}, 24 \mathrm{~h}$ and 6 days of $\mathrm{K}^{+}$deprivation. After six days of growth in the absence of $\mathrm{K}^{+}$, the remaining seedlings were transferred to one-fourth strength Hoagland solution containing $\mathrm{K}^{+}$and sampled after $30 \mathrm{~h}$. Control plants were maintained in one-fourth Hoagland solution containing $\mathrm{K}^{+}$ and sampled at the corresponding time points. Five representative seedlings were harvested at each time point, and their roots were pooled for RNA extraction. The experiment was performed in triplicate, and in all assays, three independent transgenic lines were used (namely 6, 17 and 18).

Relative GUS transcript levels in treated and control roots of the transgenic tobacco lines, sampled at the indicated times, were assessed by qPCR as described above. For this, the following primer pair was used: qRTGUSF (5'TCTACTTTACTGGCTTTGGTCG3') and qRTGUSR (5'CGTAAGGGTAATGCGAGGTAC3'). In this case, the tobacco elongation factor gene was used for normalization (primers 5'TCTGATCCATTGTTGGAT GCTGG3' and 5'TTCAAACCCTTCCTCTTGCG3'). A gene encoding a potassium transporter from $N$. rustica (NrHAK1), known to be responsive to potassium starvation (Guo et al. 2008), was used as positive control in 
the deprivation assays (primers 5'GCAGAGGATATT CAACATTCAG3' and 5'CCACCTTCACCATTATCA TCAG3').

\section{Search for putative cis-regulatory elements}

Searches for the presence of putative cis-regulatory elements within the amplified promoter region were performed using the PlantCARE (Lescot et al. 2002), and PLACE (Higo et al. 1999) databases. The transcriptional start site (TSS) prediction program (Shahmuradov et al. 2005) available at Softberry (http://www.softberry. com) was used to predict the TSS position of the studied gene. Further comparative analysis was performed using the promoter regions ( $2 \mathrm{~kb}$ from the ATG) of the $A$. thaliana (AtHAK5; At4g13420) and Populus trichocarpa (PtHAK5.1; POPTR_0001s03680) orthologs of EgHAK5. The corresponding sequences were obtained from the Arabidopsis Information Resource (TAIR; http://www. arabidopsis.com) and Phytozome, respectively.

\section{Results}

\section{Selection of the EST candidate and sequence analysis}

In searching for tissue-specific candidates within a set of $E$. grandis EST with categorized organ/tissue expression profiles (Vicentini et al. 2005), we identified an EST (EGMCRT3145G04.g) predicted to be specifically expressed in eucalyptus roots. BLAST searches against the GenBank database revealed that the deduced amino acid sequence of the validated EST shares similarity to high-affinity HAK5-like potassium transporters from different plant species, including A. thaliana (AtHAK5), $P$. trichocarpa, Vitis vinifera and Juglans regia, with more than $70 \%$ amino acid identity. HAK5 belongs to the KUP/ HAK/KT family of potassium transporters and plays a prominent role in high-affinity $\mathrm{K}^{+}$uptake (reviewed in Nieves-Cordones et al. 2016a). The presence of a "K_ trans" domain (Pfam Profile PF02705) typically found in all HAK family members indicates that the identified gene (designated hereafter $E g H A K 5$ ) encodes a putative HAK5 transporter specifically expressed in Eucalyptus roots. According to the E. grandis reference genome available in Phytozome, EgHAK5 (accession Eucgr. G02011) is located on chromosome 7 and possesses 9 exons. Interestingly, a previous search in the Eucalyptus genome identified at least 30 genes encoding KUP/HAK/ KT transporters (Nieves-Cordones et al. 2016b).
Validation of the root-specific expression of the selected EST

The predicted root-specific expression of the selected EST was confirmed by qRT-PCR using a panel of five Eucalyptus organ/tissue types. As shown in Fig. 1a, the identified gene candidate was indeed exclusively expressed in Eucalyptus roots as compared to a control housekeeping gene (GAPDH) that was ubiquitously expressed. On the other hand, no expression was observed in the other vegetative and reproductive organs/ tissues analyzed.

To substantiate further the observed root-specific expression, we performed an additional qRT-PCR analysis using root samples collected at different stages of Eucalyptus growth. Corroborating the aforementioned results, the expression of the candidate gene was exclusively detected in root samples (Fig. 1b), while no expression was observed in a stem sample used as negative control. Moreover, transcript levels varied between root samples, being higher in roots from micropropagated seedlings (40 days) followed by roots from 6-month-old and 1-month-old plantlets, respectively.
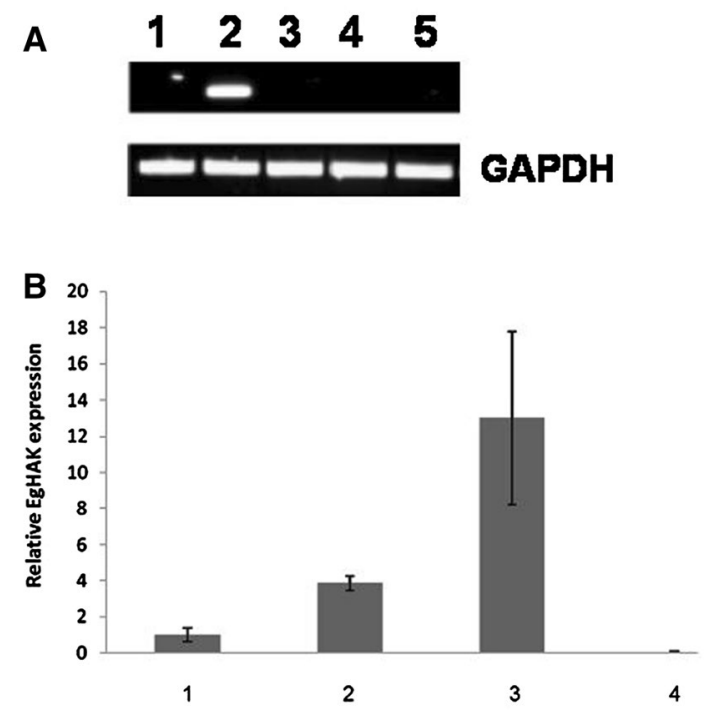

Fig. 1 Validation of the root-specific expression of the EST candidate selected in silico. a Qualitative RT-PCR analysis of the distribution of the target transcripts in different E. grandis organs/tissues. 1 pool of leaves of different ages; 2 root from 6-month-old seedlings; 3 cambium sample from 6-year-old trees; 4 stem from 6-month-old seedlings; 5 pool of flower buds and fruits. b Relative expression of the selected gene in root samples harvested from Eucalyptus of different ages. 1 roots from 1-month-old seedlings; 2 roots from 6-monthold seedlings; 3 roots from 40-day-old micropropagated seedlings; 4 stems from 40-day-old micropropagated seedlings. Average data with standard errors from three replicates is presented 


\section{EgHAK5 promoter cloning and analysis}

To ascertain whether the $E g H A K 5$ promoter would confer root-specific expression, its 5 -upstream region $(\sim 1.3 \mathrm{~kb}$ from the start codon) was amplified by GW, cloned and sequenced. The obtained sequence was subjected to the Phytozome database and confirmed to contain the 5'-upstream region of EgHAK5. As shown in Fig. 2, several potential cis-regulatory elements were identified within the $1.3 \mathrm{~kb}$ of the GW-amplified fragment. Among them, different hormone-responsive elements including an ethylene responsive motif (ERE; ATTTCAAA), a methyl jasmonic acid (MeJA) responsive motif (CGTCA), and an abscisic acid responsive element (ABRE; TAC GTG), were retrieved. The presence of two conserved motifs (TACATGT and ATAAAAT) that are over-represented in the promoter regions of a great number of rootspecific genes from Arabidopsis (Christ et al. 2013) was observed. Interestingly, the existence of two GCC-boxes (GCCGGC) previously reported within the regulatory regions of genes responsive to low potassium conditions, such as AtHAK5 (Kim et al. 2012), was equally noted. The GCCGGC motif has been previously shown to be a binding site of an ethylene-responsive transcription factor (RAP2.11) identified as a positive regulator of AtHAK5 (Kim et al. 2012). Moreover, we also identified different variants of a TGTCNN core sequence to which Auxin Response Transcription Factors (ARFs) binds. In this regard, the presence of a TGTCTC/GAGACA motif was particularly interesting, suggesting that EgHAK5 could be negatively regulated by ARF2 under adequate $\mathrm{K}^{+}$supply as recently reported for AtHAK5 (Zhao et al. 2016). Finally, a potential TATA box was located 27 bp relative to the putative TSS predicted in silico.

To gain further insights into the regulatory features of EgHAK5, we performed a comparative analysis between its 5 -upstream region ( $2 \mathrm{~kb}$ from the ATG) and those of the orthologous genes AtHAK5 and PtHAK5.1 (Fig. 3). This analysis revealed that EgHAK5 and AtHAK5 share common cis-regulatory elements involved in root-specific expression and in low- $\mathrm{K}^{+}$responsiveness in their promoters, thus suggesting a similar transcriptional regulation. Most important, all these elements, with the exception of one root-specific motif (ATAAAAT), were spatially distributed along the $1.3 \mathrm{~kb}$ fragment of the EgHAK5 promoter amplified by GW, indicating that it carries the main cis-elements required for proper activity. This is in line with the finding that a $1.25 \mathrm{~kb}$ fragment of the AtHAK5 promoter (extending from the ATG) was sufficient to direct root-specific expression in planta (Hong et al. 2013). On the other hand, the PtHAK5.1 promoter showed a divergent pattern and was enriched with a regulatory element (ATAAAAT) involved in root-specific expression. In fact, according to the expression data available at Phytozome, PtHAK5.1 is highly expressed in roots.

\section{Analysis of the $E g H A K 5$ promoter activity}

To check whether the isolated genomic fragment contained promoter activity, an expression cassette harboring a promoter-GUS fusion was constructed and stably introduced into tobacco. Tobacco was chosen due to the technical difficulties generally associated with Eucalyptus regeneration (Dobrowolska et al. 2017). Promoter-driven expression patterns were subsequently assessed by histochemical GUS assays on intact seedlings from three independent transgenic tobacco lines (namely 6,17 and 18). As a result, GUS staining was consistently observed in the vasculature of all seedlings analyzed. In this regard, the GUS signal was detected along the leaf-veins (Fig. 4a, b, c) and was prominent in vascular tissues along the root (Fig. 4d, e) and the hypocotyl (Fig. 4g). Absence of GUS expression in nonvascular tissues was evident. Cross-sections of the stained roots revealed that GUS activity was mostly associated with the phloem (Fig. 4f), thus confirming confined expression in the vasculature. The observed profile contrasted with the ubiquitous pattern of GUS expression observed in tobacco seedlings transformed with a 35S:GUS cassette (not shown). Altogether, these results indicate that the cloned $1.3 \mathrm{~kb}$ fragment possesses promoter activity but is not sufficient to direct root-specific expression in developing transgenic tobacco seedlings as seen for the endogenous gene in Eucalyptus.

\section{EgHAK5 promoter is responsive to $\mathrm{K}^{+}$starvation}

Since genes encoding HAK5 transporters are reported to be up-regulated under low $\mathrm{K}^{+}$conditions, we decided to test whether the cloned $E g H A K 5$ promoter region is responsive to $\mathrm{K}^{+}$deprivation. For this, seedlings of the investigated transgenic lines were submitted to $\mathrm{K}^{+}$starvation and GUS expression was monitored by qRT-PCR (Fig. 5). The effectiveness of the imposed stress was first confirmed by the induced expression of a tobacco gene (NrHAKl) known to be responsive to $\mathrm{K}^{+}$starvation (3.5-fold induction after 6 days).

In all transgenic lines analyzed, $\mathrm{K}^{+}$deprivation increased GUS expression reaching a maximum after $24 \mathrm{~h}$ of starvation, and declining from peak levels after 6 days (Fig. 5). However, compared to the nonstarved control, the detected induction was statically significant only for transgenic lines 6 (2.3-fold induction) and 17 (2.05-fold induction). Potassium resupply for $30 \mathrm{~h}$ caused no significant alterations in reporter expression, with the only exception of line 17, in which a significant up-regulation of GUS expression was detected (3.44-fold induction). This was unexpected, 


\begin{tabular}{|c|c|}
\hline $\begin{array}{c}\text { ERE } \\
\text { TACCGTGAAGATCAATGGTATACTTTGTTGTAGTGTGATAG TTTGAAATGTTTGGAGTA }\end{array}$ & -1201 \\
\hline 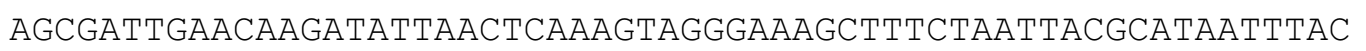 & -114 \\
\hline 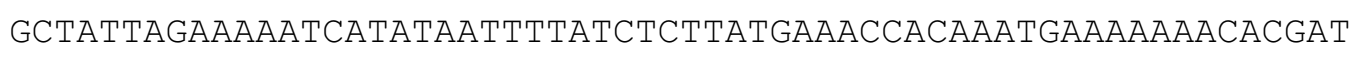 & $-10 \varepsilon$ \\
\hline 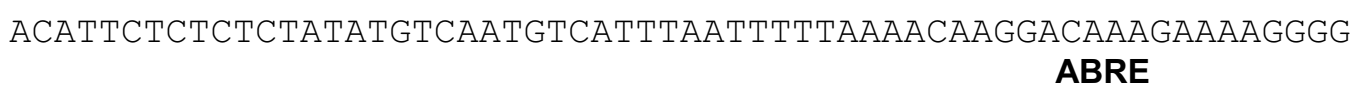 & -1 \\
\hline GAAAACATTTTTTTGGGGGGGACGCGCGATGATTATTGGGGCCGGCACGTAAGATCCCTA & -961 \\
\hline GAGAATAGCTCAATCAATCACAGTTAATTAATTTTGATAAAATAATTTCATATAАTAАCA & -9 \\
\hline TGAAGTGGGGCCTTTTTCCCAAAATATAACTAAAAATGGACTTCGTCCTAGATAAAGTCT & -841 \\
\hline TGAAGAGGCAAATTTAATCTCAAATAAGACCTTAAATTGACAAGCCGACCAAAACTTCAC & -781 \\
\hline TAGTCGATGAACATGTGTGACTCACAGGACGCTTGGCAAAACATGACTAGGGCATTTTCA & -721 \\
\hline 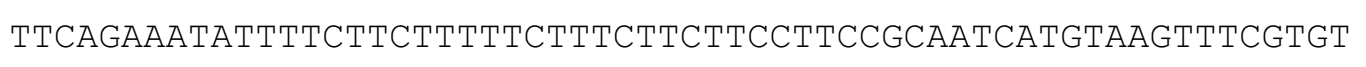 & -661 \\
\hline GCTCTTTGTCTGACCGGGCAAACTAAGTTTGTCATTTGAAAATATTATTTGAGACAAGAT & -601 \\
\hline 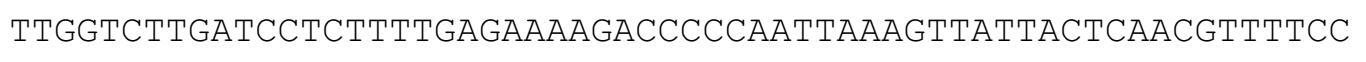 & -541 \\
\hline CCGGGTTCTACCCTTAGCTGTGATCAACTTGCACTTTTCCTTATTGGGCTTTCTTTCTTT & -481 \\
\hline СTTCTGAATAGAACCTTGGAACTAAGAAAAATACATGTCATTATTGGAAACGCCCCTTAA & -421 \\
\hline AGgAAAGgGGAAAAAAAATGTAGTTAATAGTAGTTAAATTAGTAAGAGATAAGTTTAGAC & -361 \\
\hline AGTTGACTCATGAGAAAGTACATAGTTAAATCTATCTCAGCTAGCATATGTGGAAAGCGA & -301 \\
\hline CAAAAGCGCGCAACTTGCCAAGTCGACCGATGGGGCCTAGTCACCTGAATAGAAATCCTT & -241 \\
\hline TATACTGATCCTATAAGCGAAACAAACACGTCTCACCGACAAAAAAAAAAAAАGTCAAAT & -181 \\
\hline тTTTTGAGATAAACAAACCTCAАTGATCACСтTTTTTCCCATTAAACAGTTC & -121 \\
\hline TTGTACAGCCGAATGTGCGGTTAAAGCGTCAGGACTCTCGAAGCTC $\underline{\underline{\mathbf{T A T A A A A G T G C G G A A}}}$ & -6 \\
\hline ССАТСССТTCACGTTGÄGAGAAAGGAACAGAGCGAAAAAAGGCCGGCGAGGA & -1 \\
\hline ATGGCGGAGAGGAAGGTGGTGGCGCTGGAG & +3 \\
\hline
\end{tabular}

Fig. 2 Nucleotide (nt) sequence of the cloned EgHAK 5'-flanking region. Numbers indicate the positions relative to the translational start codon ATG (marked in bold) with the adenine assigned as position +1 . The putative TSS ( a bold and underlined) is indicated. The potential TATA-box is double-underlined and in bold. The ciselements involved in hormone response (with corresponding names given above) are marked in bold italic. The modified GCC-boxes
(GCCGGC) previously identified in the promoter regions of genes responsive to low potassium conditions and recognized by RAP2.11 are indicated on a gray background. The sequences of two elements associated with root-specific expression in plants are indicated by black boxes, while the one (TGTCTC/GAGACA) recognized by ARF2 is on a dark gray background and underlined 

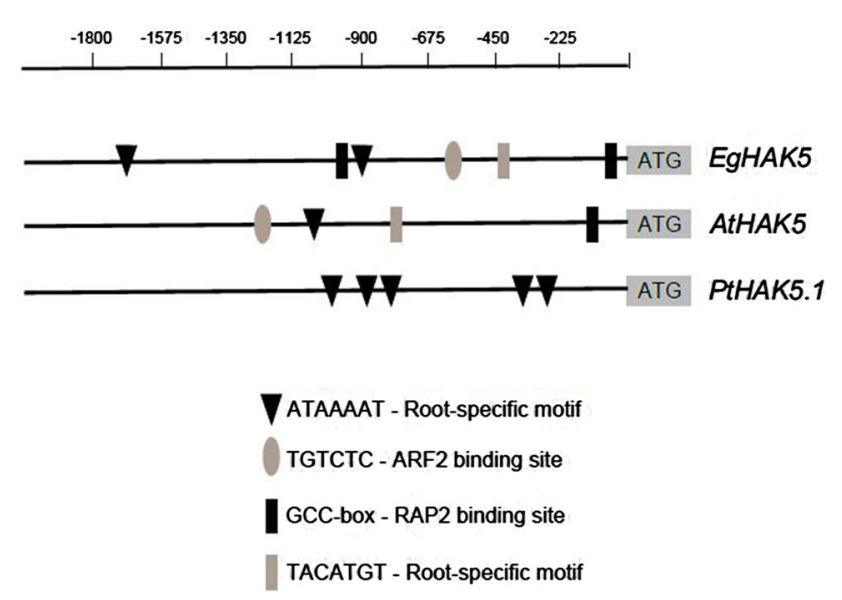

Fig. 3 Schematic representation of the putative root-specific cisregulatory motifs and RAP2.11 and ARF2 binding sites found in the promoter regions ( $2 \mathrm{~kb}$ upstream from the start codon) of $E g H A K 5$, AtHAK5 and PtHAK5.1. The symbols corresponding to each annotated cis-element are depicted

especially because HAK5 genes are reported to be upregulated under low $\mathrm{K}^{+}$supply (Ahn et al. 2004; Gierth et al. 2005; Yang et al. 2014), and might be a consequence of positional insertion effects. Despite this atypical result, the overall data indicate that $\mathrm{K}^{+}$depletion can activate the EgHAK5 promoter, which is in line with the described low
$\mathrm{K}^{+}$-inducible expression of other plant HAK5 genes (Ahn et al. 2004; Gierth et al. 2005; Alemán et al. 2009). This is also supported by the presence within the EgHAK5 promoter of two modified GCC-boxes shared with the promoter sequences of genes known to be responsive to low $\mathrm{K}^{+}$conditions such AtHAK5 (Kim et al. 2012). Interestingly, no evident alterations in histochemical GUS localization were observed in seedlings grown under $\mathrm{K}^{+}$deprivation (not shown) as previously observed for AtHAK5 (Gierth et al. 2005; Hong et al. 2013).

\section{Discussion}

In this study, we present data on the functional characterization of the promoter region of a Eucalyptus gene showing root-specific expression. This gene (named EgHAK5), predicted to encode a high-affinity HAK5-like potassium transporter, was selected within a set of Eucalyptus ESTs with defined organ/tissue expression patterns (Vicentini et al. 2005), and its supposed root-specific expression was validated using qualitative and quantitative RT-PCR.

However, although validated as a root-specific gene in Eucalyptus, the cloned EgHAK5 promoter fragment was unable to drive root-specific expression in transgenic tobacco plants. In this regard, despite the presence
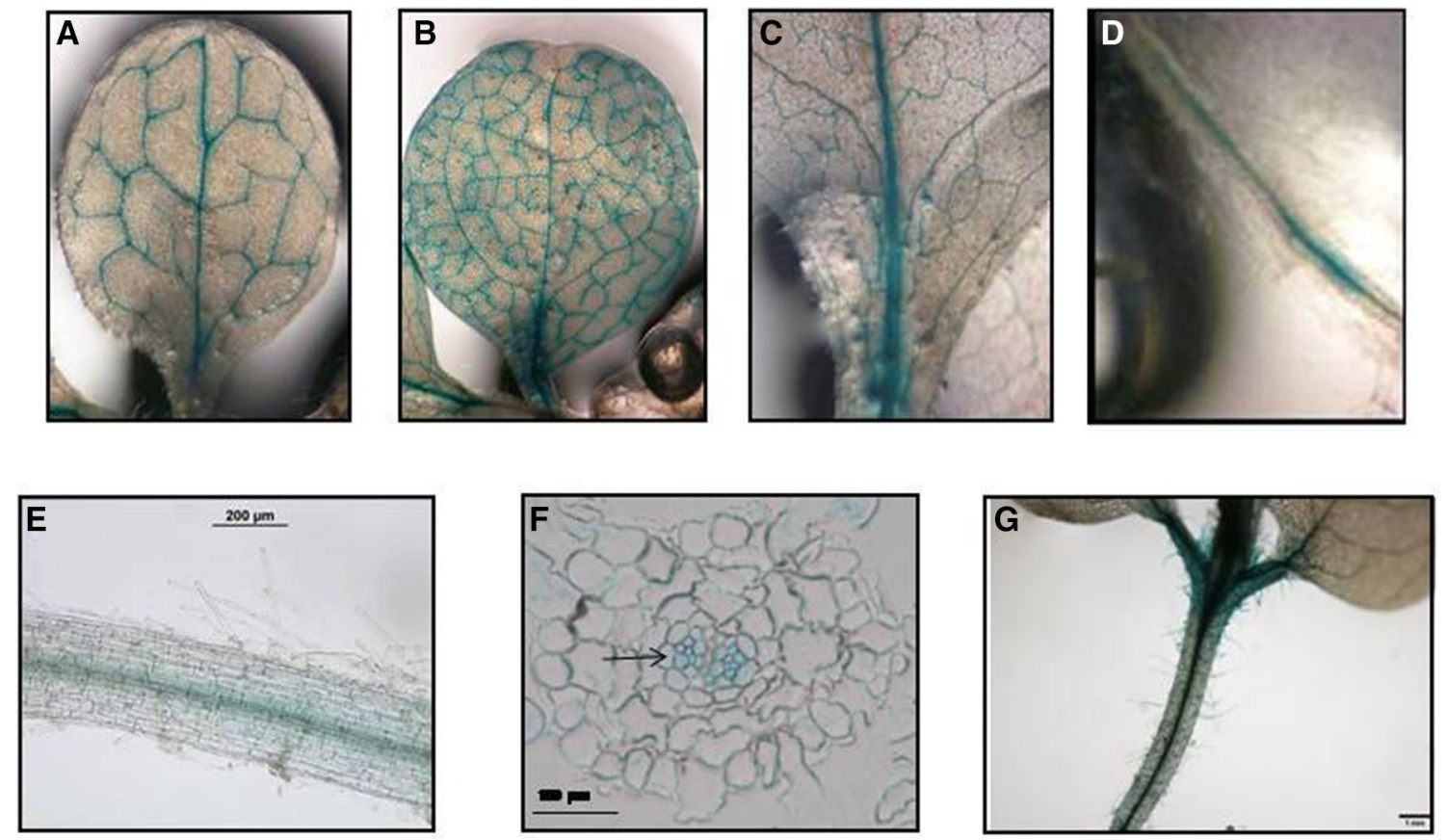

Fig. 4 Histochemical localization of GUS activity in seedlings of a representative transgenic tobacco line (06) harboring the EgHAK promoter:GUS expression cassette. a and b Leaf samples showing GUS staining at leaf-veins. c Higher magnification of a leaf petiole. d and e Root samples with GUS staining in the vascular tissues. $\mathbf{f}$ Cross-section of a stained root showing GUS signal in the phloem cells (arrow). $\mathbf{g}$ GUS staining in the vasculature of the hypocotyl of a 25 day-old seedling 


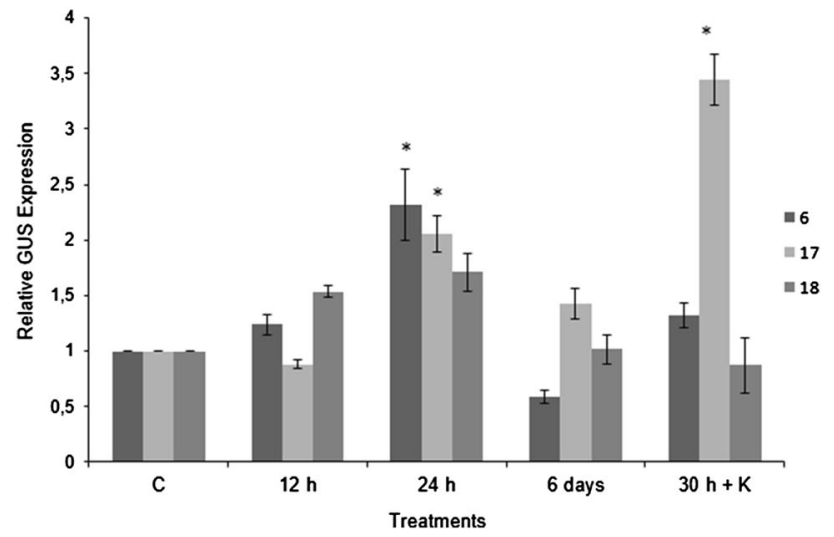

Fig. 5 Expression of the GUS reporter gene in the roots of transgenic tobacco submitted to $\mathrm{K}^{+}$starvation. Samples were collected at different times $\left(12 \mathrm{~h}, 24 \mathrm{~h}\right.$, and 6 days) after $\mathrm{K}^{+}$deprivation. After 6 days exposed to $\mathrm{K}^{+}$-free hydroponic solution, $\mathrm{K}^{+}$was resupplied and seedling roots were collected after $30 \mathrm{~h}(30 \mathrm{~h}+\mathrm{K})$. Each time point represents the average data with standard errors from three biological replicates $(* p<0.05)$. Three independent lines $(6,17$ and 18$)$ were analyzed. The expression under control condition (C) was arbitrarily set to 1

of two cis-elements (TACATGT and ATAAAAT) known to be functionally relevant for proper expression in roots (Christ et al. 2013), EgHAK5 promoter-derived GUS staining, although restricted to vascular tissues, was histochemically detected in the entire seedling. Intriguingly, the GW-amplified EgHAK5 promoter fragment shares common regulatory elements with AtHAK5, which is reported to be expressed in roots (Gierth et al. 2005; Hong et al. 2013). In a previous study, the activity of a longer version of the AtHAK5 promoter $(2.0 \mathrm{~kb}$ from the ATG) was shown to be more pronounced in the epidermis of main and lateral roots and less intense in the vasculature (Gierth et al. 2005). However, after $\mathrm{K}^{+}$resupply, GUS staining was detected only in the root vasculature. On the other hand, a shorter version of the AtHAK5 promoter ( $1.25 \mathrm{~kb}$ from the ATG) with almost the same size of the tested EgHAK5 promoter $(1.3 \mathrm{~kb})$ was able to drive GUS expression in the elongating region of primary and lateral roots only under $\mathrm{K}^{+}$deprivation (Hong et al. 2013). Likewise, the promoter of a rice HAK 5 transporter (OsHAK5) directed preferential reporter expression in the root and root-shoot junction under $\mathrm{K}^{+}$deprivation (Yang et al. 2014). Although requiring further investigation, the inability of the cloned EgHAK5 promoter to confer root-specific expression could be attributed to the lack of additional tissue-specific regulatory elements or to the absence in tobacco of a Eucalyptus-specific transacting factor. Emphasizing the complex regulatory network involved in the control of $H A K$ gene expression, Hong et al. (2013) reported that, apart from RAP2.11 and
ARF2, at least four additional transcription factors can bind to the AtHAK5 promoter and activate its expression under $\mathrm{K}^{+}$starvation.

Our results also indicate that the activity of the EgHAK5 promoter is induced by $\mathrm{K}^{+}$starvation, a feature that is in line with the known low $\mathrm{K}^{+}$-inducibility of HAK5 genes from other plant species (Ahn et al. 2004; Gierth et al. 2005; Alemán et al. 2009). The AtHAK5 promoter, for example, triggers an induction of GUS expression under low $\mathrm{K}^{+}$supply (Gierth et al. 2005). Compared to AtHAK5 that is up-regulated after $48 \mathrm{~h}$ of $\mathrm{K}^{+}$of starvation (Gierth et al. 2005), here we show that a $24 \mathrm{~h} \mathrm{~K}^{+}$-deprivation period was sufficient to activate the EgHAK5 promoter, thus pointing to a rapid transcriptional response. Previous studies have shown that AtHAK5 is positively and negatively regulated by the transcription factors RAP2.11 and ARF2, respectively (Kim et al. 2012; Zhao et al. 2016). The mechanistic reason for the fine-tuned regulation of AtHAK5 has been associated with energy saving under $\mathrm{K}^{+}$-sufficient conditions (Zhao et al. 2016). In this respect, the presence of RAP2.11 and ARF2 binding sites within the EgHAK5 promoter suggests that its transcription could be modulated by both regulatory factors through an ethylene and/ or auxin signaling pathways.

As outlined in the introduction, the availability of appropriate regulatory sequences to attain inducible and organ/tissue-specific expression in trees is still limited. Although further work is required to validate its ability to drive root-specific expression in Eucalyptus, the EgHAK5 promoter represents a valuable tool to direct inducible transgene expression to vascular tissues. In this regard, Lauvergeat et al. (2002) have demonstrated that the elements required for vascular expression are conserved among woody and herbaceous plant species, what would increase the biotechnological application of the EgHAK5 promoter. Accordingly, Creux et al. (2008) observed conserved patterns of cis-regulatory elements between orthologous cellulose synthase genes from Eucalyptus, Arabidopsis and Populus, with functional conservation of their activity. Hence, the availability of the EgHAK5 promoter will be useful to further investigate whether specific expression patterns mediated by tissue-specific promoters are maintained across different plant species.

Acknowledgements This work was supported by grants from FAPESP (Processo 03/00724-7) and CNPq (476149/04-5 and 558413/08). We thank Suzano Papel e Celulose and Fibria for providing the biological materials. We thank Mariana Arcuri for her precious help with the GUS staining assays. We are also very grateful to Dr. Anne-Lise Haenni for her careful and critical reading of the manuscript. JPB was a CNPq-PNPD post-doc fellowship recipient. CSC, CRL and ASS were FAPESP fellowship recipients. 
Author contributions Conceived and designed the experiments: CSC, FTS, JPB and IGM; performed the experiments: CSC, CRL, ASS and JPB; analyzed the data: CSC, JPB and IGM; wrote the paper: IGM.

\section{References}

Aggarwal D, Kumar A, Reddy MS (2015) Genetic transformation of endo-1,4- $\beta$-glucanase (Korrigan) for cellulose enhancement in Eucalyptus tereticornis. Plant Cell Tiss Organ Cult 122:363-371

Ahn SJ, Shin R, Schachtman DP (2004) Expression of KT/KUP genes in Arabidopsis and the role of root hairs in $\mathrm{K}^{+}$uptake. Plant Physiol 134:1135-1145

Alemán F, Nieves-Cordones M, Martinez V, Rubio F (2009) Differential regulation of the HAK5 genes encoding the high-affinity $\mathrm{K}^{+}$ transporters of Thellungiella halophila and Arabidopsis thaliana. Environ Exp Bot 65:263-269

Baghdady A, Blervacq AS, Jouanin L, Grima-Pettenati J, Sivadon P, Hawkins S (2006) Eucalyptus gunnii CCR and CAD2 promoters are active in lignifying cells during primary and secondary xylem formation in Arabidopsis thaliana. Plant Physiol Biochem 44:674-683

Christ A, Maegele I, Ha N, Nguyen HH, Crespi MD, Maizel A (2013) In silico identification and in vivo validation of a set of evolutionary conserved plant root-specific cis-regulatory elements. Mech Dev 130:70-81

Creux NM, Ranik M, Berger DK, Myburg AA (2008) Comparative analysis of orthologous cellulose synthase promoters from Arabidopsis, Populus and Eucalyptus: evidence of conserved regulatory elements in angiosperms. New Phytol 179:722-737

de la Torre F, Rodríguez R, Jorge G, Villar B, Álvarez-Otero R, GrimaPettenati J, Gallego PP (2014) Genetic transformation of Eucalyptus globulus using the vascular-specific EgCCR as an alternative to the constitutive CaMV35S promoter. Plant Cell Tiss Organ Cult 117:77-84

Dobrowolska I, Andrade GM, Clapham D, Egertsdotter U (2017) Histological analysis reveals the formation of shoots rather than embryos in regenerating cultures of Eucalyptus globulus. Plant Cell Tiss Organ Cult 128:319-326

Fiorani Celedon PA, de Andrade A, Meireles KG, Gallo de Carvalho MC, Caldas DG, Moon DH, Carneiro RT, Franceschini LM, Oda S, Labate CA (2007) Proteomic analysis of the cambial region in juvenile Eucalyptus grandis at three ages. Proteomics 7:2258-2274

Gierth M, Mäser P, Schroeder JI (2005) The potassium transporter AtHAK5 functions in $\mathrm{K}(+)$ deprivation-induced high-affinity $\mathrm{K}(+)$ uptake and AKT1 $\mathrm{K}(+)$ channel contribution to $\mathrm{K}(+)$ uptake kinetics in Arabidopsis roots. Plant Physiol 137:1105-1114

Goicoechea M, Lacombe E, Legay S, Mihaljevic S, Rech P, Jauneau A, Lapierre C, Pollet B, Verhaegen D, Chaubet-Gigot N, Grima-Pettenati J (2005) EgMYB2, a new transcription activator from Eucalyptus xylem, regulates secondary cell wall formation and lignin biosynthesis. Plant J 43:553-567

Guo Z, Yang Q, Wan X, Yan P (2008) Functional characterization of a potassium transporter gene NrHAK1 in Nicotiana rustica. J Zhejiang Univ Sci B 9:944-952

Hernandez-Garcia CM, Finer JJ (2014) Identification and validation of promoters and cis-acting regulatory elements. Plant Sci 217-218:109-119

Higo K, Ugawa Y, Iwamoto M, Korenaga T (1999) Plant cis-acting regulatory DNA elements (PLACE) database. Nucleic Acids Res 27:297-300

Hoagland DR, Arnon DI (1950) The water culture method for growing plants without soil. California Agricultural Experiment Station, Berkeley, p 347
Hong JP, Takeshi Y, Kondou Y, Schachtman DP, Matsui M, Shin R (2013) Identification and characterization of transcription factors regulating Arabidopsis HAK5. Plant Cell Physiol 54:1478-1490

Horsch RB, Fry JE, Hoffmann NL, Eichholtz D, Rogers SD, Fraley RT (1985) A simple and general method for transferring genes into plants. Science 227:1229-1231

Jefferson RA, Kavanagh TA, Bevan MW (1987) GUS fusions: betaglucuronidase as a sensitive and versatile gene fusion marker in higher plants. Embo J 6:3901-3907

Kim MJ, Ruzicka D, Shin R, Schachtman DP (2012) The Arabidopsis AP2/ERF transcription factor RAP2.11 modulates plant response to low-potassium conditions. Mol Plant 5:1042-1057

Korimbocus J, Coates D, Barker I, Boonham N (2002) Improved detection of Sugarcane yellow leaf virus using a real-time fluorescent (TaqMan) RT-PCR assay. J Virol Meth 103:109-120

Lacombe E, Van Doorsselaere J, Boerjan W, Boudet AM, Grima-Pettenati J (2000) Characterization of cis-elements required for vascular expression of the cinnamoyl CoA reductase gene and for proteinDNA complex formation. Plant J 23:663-676

Lauvergeat V, Rech P, Jauneau A, Guez C, Coutos-Thevenot P, GrimaPettenati J (2002) The vascular expression pattern directed by the Eucalyptus gunnii cinnamyl alcohol dehydrogenase EgCAD2 promoter is conserved among woody and herbaceous plant species. Plant Mol Biol 50:497-509

Lescot M, Dehais P, Thijs G, Marchal K, Moreau Y, Van de Peer Y, Rouze P, Rombauts S (2002) PlantCARE, a database of plant cisacting regulatory elements and a portal to tools for in silico analysis of promoter sequences. Nucleic Acids Res 30:325-327

Livak K, Schmittgen TD (2001) Analysis of relative gene expression data using real time quantitative PCR and the $2^{-\Delta \mathrm{Ct}}$ method. Methods 25:402-408

Lu S, Li L, Yi X, Joshi CP, Chiang VL (2008) Differential expression of three eucalyptus secondary cell wall-related cellulose synthase genes in response to tension stress. J Exp Bot 59:681-695

MacMillan CP, Taylor L, Bi Y, Southerton SG, Evans R, Spokevicius A (2015) The fasciclin-like arabinogalactan protein family of Eucalyptus grandis contains members that impact wood biology and biomechanics. New Phytol 206:1314-1327

Murashige T, Skoog F (1962) A revised medium for rapid growth and bioassays with tobacco cultures. Physiol Plant 15:473-497

Nieves-Cordones M, Martínez V, Benito B, Rubio F (2016a) Comparison between Arabidopsis and Rice for main pathways of $\mathrm{K}(+)$ and $\mathrm{Na}(+)$ uptake by roots. Front Plant Sci 5(7):992

Nieves-Cordones M, Rodenas R, Chavanieu A, Rivero R M., Martinez V, Gaillard I, Rubio F (2016b). Uneven HAK/KUP/KT protein diversity among angiosperms: species distribution and perspectives. Front Plant Sci 7:127

Ramakers C, Ruijter JM, Deprez RH, Moorman AF (2003) Assumptionfree analysis of quantitative real-time polymerase chain reaction (PCR) data. Neurosci Lett 13:62-66

Rodrigues MI, Bravo JP, Sassaki FT, Severino FE, Maia IG (2013) The tonoplast intrinsic aquaporin (TIP) subfamily of Eucalyptus grandis: characterization of EgTIP2, a root-specific and osmotic stressresponsive gene. Plant Sci 213:106-113

Severino FE, Brandalise M, Costa CS, Wilcken SR, Maluf MP, Gonçalves W, Maia IG (2012) CaPrx, a Coffea arabica gene encoding a putative class III peroxidase induced by root-knot nematode infection. Plant Sci 191-192:35-42

Shahmuradov IA, Solovyev VV, Gammerman AJ (2005) Plant promoter prediction with confidence estimation. Nucleic Acids Res 18:1069-1076

Venter M, Botha FC (2004) Promoter analysis and transcription profiling: integration of genetic data enhances understanding of gene expression. Physiol Plant 120:74-83 
Vicentini R, Sassaki FT, Gimenes MA, Maia IG, Menossi M (2005) In silico evaluation of the Eucalyptus transcriptome. Genet. Mol Biol 28:487-495

Yang T, Zhang S, Hu Y, Wu F, Hu Q, Chen G, Cai J, Wu T, Moran N, Yu L, Xu G (2014) The role of a potassium transporter OsHAK5 in potassium acquisition and transport from roots to shoots in rice at low potassium supply levels. Plant Physiol 166:945-959
Zhao S, Zhang ML, Ma TL, Wang Y (2016) Phosphorylation of ARF2 relieves its repression of transcription of the $\mathrm{K}^{+}$transporter gene HAK5 in response to low potassium stress. Plant Cell. doi:10.1105/tpc.16.00684 